\title{
Using ultrasonic waves and finite element method to evaluate through-thickness residual stresses distribution in the friction stir welding of aluminum plates
}

\author{
Seyedali Sadeghi ${ }^{1}$ \\ ${ }^{1}$ Department of Mechanical Engineering, Amirkabir University of Technology, 424 Hafez Ave.,15914, Tehran, Iran. \\ sadeghi1368@aut.ac.ir
}

Mehdi Ahmadi Najafabadi ${ }^{2}$

${ }^{2}$ Department of Mechanical Engineering, Amirkabir University of Technology, 424 Hafez Ave.,15914, Tehran, Iran. ahmadin@aut.ac.ir

Yashar Javadi ${ }^{3 *}$

${ }^{3}$ Department of Mechanical Engineering, Semnan Branch, Islamic Azad University, Semnan, Iran.

( ${ }^{*}$ Corresponding author's e-mail: yasharejavadi@yahoo.com; Tel: +98 9124402303, Fax: +98 2313354030.$)$

Mohammadjavad Mohammadisefat ${ }^{4}$

${ }^{4}$ Department of Mechanical Engineering, Amirkabir University of Technology, 424 Hafez Ave.,15914, Tehran, Iran.

m.j.mohammadi@aut.ac.ir

\begin{abstract}
The main goal of this study is ultrasonic measuring of through-thickness residual stresses in friction stir welding of aluminum plates. A 3D thermo-mechanical finite element analysis is used to evaluate residual stresses caused by friction stir welding of 5086 aluminum plates. The finite element (FE) model has been validated by the hole drilling method. Residual stresses obtained from the FE analysis is then compared with those obtained from ultrasonic stress measurement. The ultrasonic measurement technique is based on acoustoelasticity law which describes the relation between the acoustic waves and the stress of material. The ultrasonic stress measurement is accomplished by using longitudinal critically refracted $\left(L_{C R}\right)$ waves which are propagated parallel to the surface within an effective depth. Through-thickness distribution of longitudinal residual stresses is evaluated by employing the $L_{C R}$ waves produced by four different frequencies ( $1 \mathrm{MHz}, 2 \mathrm{MHz}, 4 \mathrm{MHz}$ and $5 \mathrm{MHz}$ ) of ultrasonic transducers. Utilizing the FE analysis along with the $L_{C R}$ method (known as $F E L_{C R}$ method), the through-thickness distribution of longitudinal residual stress could be achieved. The comparison between ultrasonic and FE results show an acceptable agreement, hence the $F E L_{C R}$ method could be successfully applied on the FSW plates. It has been concluded that the longitudinal residual stresses of aluminum plates joined by friction stir welding can be evaluated by using the $F E L_{C R}$ method. The good potential of $F E L_{C R}$ as a nondestructive method is also confirmed in through-thickness stress measurement of aluminum plates.
\end{abstract}




\section{Keywords: Friction Stir Welding; Finite Element Welding Simulation; Ultrasonic Stress Measurement; Welding Residual Stress; Through Thickness Stress; Acoustoelasticity.}

\section{Introduction}

The engineering properties of the structural components, particularly fatigue life, deformation, dimensional stability, corrosion resistance, and brittle fracture can be significantly influenced by residual stresses. Residual stresses can be defined as remaining stresses inside the material after manufacturing, in the absence of external loads or thermal gradients. Welding is an essential production process in the industry which produces residual stresses at a remarkable level. Welding residual stresses are caused by non uniform thermal expansions and solidifications during the welding processes.

Residual stresses evaluation is an essential stage in the design of structures and also in the estimation of their reliability under real service conditions. Over the years, different methods have been presented to measure residual stress for different types of equipments in order to obtain reliable assessment while the majority of these methods are described by Rossini et al [1]. The residual stresses are measured by Olabi and Hashmi [2] where it has been shown that the post-weld heat-treatment leads to reducing residual stresses by about $70 \%$. The welding residual stresses are also measured by them in different studies [3-6] to investigate the influence of various parameters on the residual stresses of welded structures.

The welding residual stresses may be available in all of the welding processes including both fusion and solid state welding processes. The solid state welding processes do not involve the melting of the materials being joined; instead, the weld is formed by producing coalescence at temperatures essentially below the melting point of the base materials, without the addition of brazing filler metal. Friction stir welding (FSW) is a solid state welding process patented in 1991 at TWI [7], in which a rotating tool (pin) is first embedded into the adjoining edges of the plates to be welded with a pre-determined tilt angle, and then moved all along the welded joint. Such a pin produces frictional heating along with plastic deformation in the welding zone while no melting of material is created. As the tool moves, the material is forced to flow around the tool in a relatively complex flow pattern which has been described in several literatures [8-10].

More recently, a few papers focused on the analysis of residual stress produced by the FSW. Bussu and Irving [11] showed that the fatigue life is slightly improved by presence of the residual stresses; however they considered deformations induced by the residual stresses that restrict application of the FSW process in manufacturing production. Peel et al. [12] employed the X-ray measurements to investigate the tool feed rate effect on distribution of residual stresses caused by the friction stir welding of 5083 aluminum plates. Similar studies were developed by Staron et al. [13] and Preve'y et al. [14] on the friction stir welding of aluminum.

Nondestructive measurement of residual stresses is necessary to optimize the structures' design and improve the mechanical strength. There is no reliable nondestructive method that brings complete satisfaction in the in-situ stress monitoring of the welded equipments. Material, geometry, surface quality, cost, and accuracy of the measurement are some of the parameters that must be considered in choosing a proper method for stress measurement. The development of nondestructive methods like X-ray diffraction and the ultrasonic technique along with semi-destructive methods like incremental hole-drilling technique is inevitable for stress measurement and health monitoring of the equipments.

Ultrasonic stress measurement is founded on the linear relation between the velocity of the ultrasonic wave and the material stress. This correlation, within the elastic limit, is called as acoustoelastic effect which shows that flight time of the ultrasonic wave changes linearly with the stress. In 1967, Crecraft [15] showed that the acoustoelastic effect can be employed for stress measurement of engineering materials. The longitudinal critically refracted $\left(L_{C R}\right)$ wave is a longitudinal ultrasonic wave which can be propagated parallel to the surface. It has been proved by Egle and Bray [16] that sensitivity of the $L_{C R}$ waves to the stress is highest among the other types of ultrasonic waves. Tang and Bray [17] utilized the $L_{C R}$ waves to measure the stresses higher than yield strength and also studied plastic deformation effect on the velocity variations of waves in 4140 steel. The $L_{C R}$ waves were used to evaluate the bending stress in steel plates and bars by Bray and Tang [18]. They employed two different testing frequencies ( $2.25 \mathrm{MHz}$ and $5 \mathrm{MHz}$ ) and compared the results. The $L_{C R}$ measurement was accomplished in immersion mode by Belahcene and Lu [19] to measure welding residual stresses of S355 steel plate. They used hole-drilling method to validate the 
ultrasonic results. Palanichamy et al [20] evaluated the residual stresses in austenitic stainless steel weld joints using ultrasonic technique.

The main goal of this research is feasibility study for nondestructive stress measurement to obtain the through-thickness distribution of longitudinal residual stresses. The ultrasonic $L_{C R}$ waves are employed to measure the residual stresses caused by the friction stir welding (FSW) of aluminum plates. The FSW process is also modeled by using the finite element method (FEM) while the distribution of longitudinal residual stresses obtained from the FEM will be validated by hole-drilling measurement. The longitudinal residual stresses calculated by FEM are then compared with those measured by ultrasonic technique while a good agreement is observed. The combination of the FE welding simulation and the ultrasonic $L_{C R}$ method (known as $F E L_{C R}$ by Javadi et al [21]) is confirmed to have enough potential for non-destructive evaluation of residual stresses through the thickness of aluminium FSW joints.

\section{Theoretical Background}

\section{1. $L_{C R}$ method}

Different ultrasonic configurations can be used for residual stresses measurements performed by the $L_{C R}$ waves. As a common experimental setup, longitudinal waves are produced at the first critical angle by a transmitter transducer, then propagate parallel to surface of the tested material and finally are detected by receiver transducers. Two transducers are utilized as the receivers to decrease the environmental effects, like ambient temperature, on the wave velocity. More experimental details of $L_{C R}$ waves can be found in several previous studies [21-27]. The relation between measured travel-time change of $L_{C R}$ wave and the corresponding uniaxial stress is derived by Egle and Bray [16] to be:

$$
\Delta \sigma=\frac{E}{L t_{0}}\left(t-t_{0}\right)
$$

In Eq. (1), $\Delta \sigma$ is stress change, $E$ is the elastic modulus and $L$ is the acoustoelastic constant for longitudinal waves propagated in the direction of the applied stress field. The acoustoelastic constant should be measured by the uniaxial tensile test performed on the samples extracted from the tested material. Also, $t$ is the experimental travel-time which would be measured on the tested material; $t_{0}$ is travel-time for a homogeneous, isotropic, stress-free sample at the room temperature. With knowledge of the weld induced change in travel-time along with the measured acoustoelastic constant, the stress variations produced by the welding process can be calculated. For a fixed distance of transducers, the travel time of the $L_{C R}$ wave decreases because of compressive stress and increases in tensile stress field. The residual stress in a subsurface layer is also measurable by using different frequencies of the transducers which produce the $L_{C R}$ waves while the depth of layer is related to the ultrasonic wave frequency, often exceeding a few millimetres.

\subsection{Finite Element Welding Simulation}

\subsubsection{FE model of FSW}

The fully 3D FEM model, used in this study, is a thermo-mechanically coupled analysis which assumes a rigid-viscoplastic material behavior. This model represents the plate seam (abutting edges) as a continuum which avoids the numerical instabilities caused by discontinuities at the edge of the two plates.

The commercial FEA software DEFORM-3D [28], a Lagrangian implicit code designed for metal forming processes, is employed to model the FSW process. The use of an implicit code versus an explicit one is a sort of inevitable choice being the latter better suited in order to correctly estimate temperature history and stress states.

As far as the mechanical analysis is regarded, a rigid-viscoplastic material model along with the Von Mises yield criterion and associated flow rule is used. The rigid-viscoplastic finite element formulation is founded on the variational approach. The actual velocity field is determined by the stationary value related to the variation of governing functional $\pi$, as described in detail in [29]: 


$$
\delta \pi=\int_{V} \bar{\sigma} \delta \dot{\bar{\varepsilon}} d V+K \int_{V} \dot{\varepsilon}_{V} \delta \dot{\varepsilon}_{V} d V-\int_{S_{F}} F_{i} \delta u_{i} d S=0
$$

In Eq. (2), $\bar{\sigma}=\bar{\sigma}(\bar{\varepsilon}, \dot{\bar{\varepsilon}}, T)$ and $\dot{\varepsilon}_{V}=\dot{\varepsilon}_{i i}$ is the volumetric strain-rate. The constant $\mathrm{K}$ should have very large positive value for incompressibility. As far as the thermal effects are considered, the temperature generated in the process has a significant influence on the mechanical response. The final thermal equilibrium equation can be expressed as:

$$
[C]\{\dot{T}\}+\left[K_{C}\right]\{T\}=\{Q\}
$$

The FSW tool is assumed to be rigid because the yield strength of the plate (i.e. aluminum alloy) is significantly lower than the yield strength of the tool (i.e. H13 steel). The workpiece is modeled by assuming rigid viscoplastic material which this assumption leads to missing the possibility of residual stresses calculation at the end of process simulation. Hence, an additional simulation by assuming the elastoplastic material model is needed to get the final residual stress state. The numerical modeling of FSW processes results in extremely long computational times due to the complex non-linearity of the mechanical analysis and the severe gradients of the main variables including strain and also strain rate around the tool action area.

During the FSW process, the workpiece is clamped at its sides which these boundary conditions are modeled in the FE analysis. The FE simulation of the FSW process is then divided into two stages: (i) sinking stage and (ii) welding (advancing) stage. In other words, FSW is modeled from its initial state (entering the pin into the aluminum plate) to the end (when the tool is removed from the clamped plates). During the sinking stage, the tool moves down vertically while it is rotating; then, during the welding stage, it moves along the welding line to join two aluminum plates. The tool is also tilted of $2^{\circ}$ angle with respect to a vertical axis. The dwelling time is considered at the end of the sinking stage to assure sufficient heating of the workpiece for the subsequent welding process.

The thermal conductivity $\mathrm{k}=120\left[\mathrm{~N} /\left(\mathrm{s}^{\circ} \mathrm{C}\right)\right]$ and thermal capacity $\mathrm{c}=2.4\left[\mathrm{~N} /\left(\mathrm{mm}^{2{ }^{\circ}} \mathrm{C}\right)\right]$ are used for the material modeling of the considered 5086 aluminum alloy. No variation of $\mathrm{k}$ and $\mathrm{c}$ with temperature is assumed to linearizes the thermal analysis which leads to better convergence. A constant interface heat exchange coefficient of $11\left[\mathrm{~N} /\left(\mathrm{mm} \mathrm{s}{ }^{\circ} \mathrm{C}\right)\right]$, is utilized for the tool-sheet contact surface. As the value of the heat transfer coefficient is regarded, a typical convection coefficient in calm air equal to $0.02\left[\mathrm{~N} /\left(\mathrm{mm} \mathrm{s}^{\circ} \mathrm{C}\right)\right]$ is selected.

As briefly described before, a rigid-viscoplastic temperature and strain rate dependent material model is used as follows:

$$
\sigma=2.03 \times 10^{3} T^{-0.488}(\dot{\bar{\varepsilon}})^{0.0027}(\bar{\varepsilon})^{0.124}[M P a]
$$

In Eq. (4), the temperature is expressed in $\mathrm{K}$ and the strain rate in $\mathrm{s}^{-1}$.

It should be noted that the material behavior is inputted in the utilized software in form of tabular data in regarding to the above assumptions and also previous literature data [30]. Eq. (4) is then obtained through linear regression just to represent the material behavior at the varying of temperature, strain and strain rate.

For the FE modeling of the workpiece, a "single block" continuum model (sheet blank without gap) is used to avoid contact instabilities due to intermittent contact produced in the plate-plate and plate-tool interfaces. In particular, a single plate is considered and the rotating tool, once completed the sinking phase, is moved forward to weld the groove left behind the pin as it advances along the welding line. 
A basic finite element model related to the rigid-viscoplastic material simulation is shown in Fig. 1a. The plate blank is meshed with about 30,023 tetrahedral elements while 14,943 tetrahedral elements are used to mesh the tool. The selection of tetrahedral elements instead of other elements, e.g. brick elements, leads to reach more reasonable computational time. A non-uniform mesh, characterized by density windows all along the welding line and adaptive re-meshing are used in order to employ smaller elements close to the tool. A re-meshing zone (which uses finer mesh size) is identified all along the tool feed movement (Fig. 1a). Proper accuracy is achieved by increasing the number of elements around the tool pin by employing the remeshing zone. In this way, lower computational time along with more accurate convergence is assured. However, many FE models with different mesh size should be run to find the efficient mesh size showed in Fig. 1a. The other FE models showed that a coarser mesh (than the mesh size selected in the efficient model showed in Fig. 1a) leads to inaccurate results and also frequent software stops; on the other hand, a finer mesh results in unaffordable computational time without valuable improvement in the accuracy.

\subsubsection{Developed FE model}

It has been proved that finite element (FE) simulation represents a valuable aid in the FSW processes design. The determination of residual stress which leads to the prediction of welded joint fatigue life has a strategic importance for most of the applications in the aeronautical, aerospace, automotive and naval industries. However, the extremely high computational time is still an important drawback in FE simulation of FSW. Depending on the joint dimensions and process parameters, a thermomechanical coupled with elasto-plastic material behavior including the mechanical action of the tool may take weeks to be analyzed.

There is a developed approach presented by Buffa et al [31] able to effectively cut down the computational time by splitting the FSW simulation into two different parts. The process is firstly simulated from the beginning, i.e. the tool sinking, to its end, i.e. the tool removing from the workpiece, with a rigid-viscoplastic material model as described in the section 2.2.1. In this way a relatively rapid convergence is reached, although the residual stresses cannot be obtained. At the end of the first part of simulation, a new database is created by a specific routine developed and embedded in new simulation software. For each node, the temperature history experienced during the first part of simulation will be imported into the second part. The workpiece is exposed to the thermal results obtained from the previous thermo-mechanically coupled analysis with no further tool mechanical action because the residual stresses mainly depend on the temperature history experienced by the workpiece [30].

During this latter part of simulation the tool object is disabled while the boundary conditions including the actual clamping fixtures are performed. This new simulation eliminates the tool mechanical action which leads to avoiding the convergence difficulties, hence reasonable computational time could be achieved.

The final step of the FE analysis consists in cooling the weld down back to the environment temperature. The cooling step is also divided into two different sub-simulations because the workpiece is clamped to the welding machine during the initial cooling stage while the boundary constraints are released in the second phase of cooling simulation. Once the joint is separated from the welding machine, three nodes of the workpiece mesh are kept fixed in order to avoid any undesired movement. Finally, the residual stress in the workpiece could be obtained at the end of this cooling step.

In this study, separating the thermo-mechanical rigid-viscoplastic simulation from the elasto-plastic one is performed by employing the DEFORM and ABAQUS software. The data coming from the DEFORM simulation are manually transferred to a further elasto-plastic FE model using a brick type mesh in ABAQUS environment (Fig. 1b). The elasto-plastic FE model is developed with ABAQUS/Standard 6.11 [32] using the coupled temperature displacement analysis option. The plate is modeled with 4590 brick elements of uniform geometry (see Fig. 1b). These elements are characterized by eight nodes and tri-linear displacement, and temperature functions reduced integration with hourglass control. The boundary conditions of the model are defined to simulate the clamping and supporting conditions applied during the FSW process. At each node of the model, the corresponding temperature history is input (from DEFORM results) as transitory boundary condition, reproducing the thermal effects derived both the friction-forces work and deformation work. At the end of the simulation, the mechanical constraints are released to reproduce the unclamping of the joint (after the FSW process). In this way, the longitudinal residual stresses are achieved which will be used to be compared with those obtained from the ultrasonic stress measurement. 


\section{Experimental Procedures}

\subsection{Sample Description}

Two $150 * 100 * 8 \mathrm{~mm}$ plates made from 5086 aluminum are friction stir welded in butt joint geometry. The FSW tool is made from $\mathrm{H} 13$ steel which the design and dimensions is shown in the Fig. 2. The FSW is performed by using a vertical milling machine while the process parameters including feed rate, tool rotating speed and tilt angle are equal to $0.2 \mathrm{~mm} / \mathrm{s}, 400 \mathrm{rpm}$ and $2^{\circ}$ respectively. During the FSW process, the workpiece is clamped at its sides by using a steel fixture and also supported at its bottom by a back plate (Fig. 2).

\subsection{Measurement Devices}

The measurement device, shown in Fig.3, includes an ultrasonic box, computer and time of flight (TOF) measuring equipments. Also, an automatic measuring table is needed to move TOF measuring equipments with proper accuracy and stability. The automatic table is supported by 3 step motors to be able to move the transducers in 3 axes with resolution of $1 \mu \mathrm{m}$. The ultrasonic box is a portable ultrasonic testing device with sample rate of $100 \mathrm{MHz}$. The analogue to digital (A/D) converter is controlled by synchronization between the pulser signal and the internal clock. The clock works with the resolution of $1 \mathrm{~ns}$ which allows very precise measurements of the time of flight. The TOF measuring equipments include three longitudinal transducers assembled on an integrated wedge to measure the time of flight. The laser cutting (with consideration to the results presented by Eltawahni et al [33]) and CNC milling are employed to construct the wedge from poly methyl methacrylate (PMMA) material, under the trademark Plexiglas. A three-probe configuration is used, with one sender and two receivers in order to eliminate temperature effect on the travel time. Twelve transducers in four different frequencies are utilized where their nominal frequencies are $1 \mathrm{MHz}, 2 \mathrm{MHz}, 4 \mathrm{MHz}$ and $5 \mathrm{MHz}$. The $L_{C R}$ wave produced by using low frequency transducers could be penetrate more in the material, hence using different frequencies helps to evaluate residual stresses in different depths of the material. A constant pressure on the wedge is needed in order to keep constant thickness of ultrasonic couplant layer between the wedge and tested plate. The pressure is supported by using a compressor and pneumatic cylinder. There is also a couplant layer between the assembled transducer and its location in the wedge while the plastic screws support the constant pressure on the transducers.

\subsection{Determination of $L_{C R}$ Penetration Depth}

When the $L_{C R}$ technique is applied to a workpiece with limited wall thickness, the penetration depth of the $L_{C R}$ wave is expected to be a function of frequency. Since there is no reliable equation for the relation between the penetration depth and frequency, it should be measured experimentally. Four different frequencies have been used in this study to evaluate the longitudinal residual stress through the thickness of plates; hence the depth of all frequencies should be exactly measured. A variable depth groove is cut in a plate, with the same material and thickness of the tested plates, producing a barrier to physically prevent the $L_{C R}$ wave from reaching the receiver transducers. It was found that a $6.5 \mathrm{~mm}$ depth groove could completely prevent a $1 \mathrm{MHz}$ $L_{C R}$ wave to pass, which indicates that the penetration depth of such a $L_{C R}$ wave is $6.5 \mathrm{~mm}$. The penetration depths of $L_{C R}$ wave produced by $2 \mathrm{MHz}, 4 \mathrm{MHz}$ and $5 \mathrm{MHz}$ transducers are measured equal to $3.4 \mathrm{~mm}, 1.5 \mathrm{~mm}$ and $1.1 \mathrm{~mm}$ respectively.

\subsection{Evaluation of the Acoustoelastic Constants}

The standard uniaxial tensile tests are needed to evaluate the acoustoelastic constant while a changed form of Eq. (1) can be used for the calculations:

$$
L=\frac{E}{\Delta \sigma \times t_{0}}\left(t-t_{0}\right)
$$

Tensile test specimens are extracted from the workpiece and would be exposed to the tensile test while the TOF measuring equipments are assembled on the specimen in order to measure flight-time $(t)$ of the $L_{C R}$ wave. However, before starting the tensile test, the specimens should be stress relieved to measure the TOF of stress-free samples $\left(t_{0}\right)$. By using a tensile test standard machine, the tensile test $(\sigma)$ is increased step by step while the flight-time $(t)$ is measured in each step. The elastic 
modulus $(E)$ could also be measured by using the tensile test results or obtaining from the material tables. As a result, the acoustoelastic constant $(L)$ can be calculated.

In this study, the tensile test specimens are extracted from the weld and parent material (PM) to measure the $E$ and $L$ in each of these zones. The tensile test specimens are constructed with the specifications which meet the Sheet type ( 0.5 in. wide) sample presented in the ASTM: E8 standard. The results of acoustoelastic constant measurement are listed in Table 1 according to the frequency of TOF measuring transducers which are assembled on the specimen during the tensile test.

\section{3-5. Hole-drilling measurement to validate FE model}

In this study, the FE welding simulation is employed to verify the accuracy of ultrasonic stress measurement. It is also needed to prove the accuracy of FE model by using the other experimental methods. Hence, the residual stresses obtained from the FE simulation is validated by utilizing the hole-drilling measurement. The hole-drilling method is performed in three different points (as shown in Fig. 4) based on the characterizations described in ASTM: E837. This semi-destructive technique determines the stresses near the surface of a sample through the incremental drilling of a small hole, which relaxes the stresses in that location. The resultant strains are measured using a strain gauge rosette (Fig. 4) after each depth increment and the biaxial stress field is then calculated employing equations established by ASTM: E837.

\section{Results and Discussion}

\subsection{Employing the $L_{C R}$ waves and FEM to evaluate through thickness stresses of FSW}

Completing the following steps is required to reach the main goal of this study which is employing the ultrasonic waves and finite element method to evaluate residual stresses through thickness of the aluminum plates joined by friction stir welding process:

The measurement devices (section 3.2) are employed -1 to measure TOF in four different depths of the plate by using four different frequencies of transducers. The $L_{C R}$ wave is propagated parallel to the weld direction which leads to longitudinal stress measurement through the thickness of plates. Tensile test specimens are extracted from the weld -2 and parent material (PM) zones. The value $t_{0}$ is measured directly on the stress-free - 3 samples which are the tensile test specimens extracted from the weld and PM zones. The stressfree situation is created by employing stress relieving heat treatment.

The elastic modulus $(E)$ is measured by using -4 tensile test on the specimens extracted from the weld and PM zones. The acoustoelastic constant is measured by using a -5 uniaxial tensile test and Eq. (5) in the weld and PM zones separately (Table 1 ). 
By putting the results of step $1-5$ in Eq. (1), -6 longitudinal residual stresses can be calculated related to four measuring frequencies which present four distribution of longitudinal residual stress in four different depths of the plate (the depths are related to the penetration depths measured in section 3.3).

Comparing the ultrasonic stress measurement results -7 with those obtained from $\mathrm{FE}$ simulation could validate the ultrasonic stress measurement while the sE simulation is also verified by the hole-
drilling measurement.

Completing steps 1-7, which are summarized in Fig.5, will lead to through-thickness evaluation of longitudinal residual stresses in FSW process performed on the aluminum plates by employing the $L_{C R}$ waves and FEM.

\subsection{Results of finite element welding simulation}

\subsubsection{Verification of FE model}

The longitudinal residual stresses analyzed by FE simulation are compared with those obtained from the hole-drilling measurements (Fig.6) and a good agreement is achieved. This agreement can be considered as validation of FE welding simulation and prove the accuracy of FE model. Hence, the FE model could be employed in verifying the results of ultrasonic stress measurements.

\subsubsection{Residual stress analysis}

The validated FE model could be used for residual stress analysis now. The longitudinal residual stress calculated by finite element simulation is shown in Fig.7. The results are obtained for four different depths equivalent to the measured penetration depths of ultrasonic $L_{C R}$ waves (section 3.3). It can be observed that the peak of longitudinal residual stress is occurred in the advancing side (AS) of FSW. This distribution of residual stresses is because of non-symmetric temperature distribution of FSW while the maximum of welding temperature value occurs in the advancing side of the joint [31]. Furthermore, the major part of material flow occurs at the AS, hence stirred zone (SZ) stretches into the AS which cause to higher residual stresses of the advancing side [34].

It should be noticed that the $L_{C R}$ ultrasonic method measures the average of stresses in determined depths [21]. For example, the $L_{C R}$ wave produced by the $1 \mathrm{MHz}$ transducer will travel in $6.5 \mathrm{~mm}$ beneath the surface and gives the average of residual stress in this zone. Hence, the average of FE residual stresses for all the nodes located in 0-6.5 mm under the surface are calculated to be comparable with ultrasonic measurements.

The FE results show relatively homogeneous through-thickness residual stress distributions which were be expectable according to the objectives reported by Woo et al [35]. They showed that using pin in the FSW tool design lead to homogeneous throughthickness residual stress distribution while the "shoulder-only" case (no pin) exhibits residual stress variations through the thickness [34]. The pin is considered in the tool design used in this study, hence no considerable change is observed in the stress obtained from the FE simulation performed in the depth of 0-6.5 mm. However, a little difference could be observed between surface and bulk stresses while the difference is less than $20 \mathrm{MPa}$. 


\subsection{Results of ultrasonic measurements}

The longitudinal residual stresses measured by the ultrasonic method are shown in Fig.8-11 related to the $L_{C R}$ waves created by $5 \mathrm{MHz}, 4 \mathrm{MHz}, 2 \mathrm{MHz}$ and $1 \mathrm{MHz}$ respectively. The ultrasonic stress measurements are also compared with the FE results while a good agreement is achieved. The little difference between surface and bulk residual stresses (less than $20 \mathrm{MPa}$ ) could be measured by using different test frequencies. It can be also observed that the difference between ultrasonic and FE results changes with the test frequency and is less than $\pm 10 \mathrm{MPa}, \pm 7 \mathrm{MPa}, \pm 5 \mathrm{MPa}$ and $\pm 4 \mathrm{MPa}$ for the measurement performed by 5 $\mathrm{MHz}, 4 \mathrm{MHz}, 2 \mathrm{MHz}$ and $1 \mathrm{MHz}$ transducers respectively. It means that using higher test frequency lead to lesser accuracy of ultrasonic measurement. This objective was also reported by Javadi et al [21-27].

In addition to the measurement errors of ultrasonic method, the difference between FE and ultrasonic results can also be justified by different acoustic properties of AS and RS materials which can produce a complicated weld region. However, better accuracy could be achieved by decreasing the dimension of weld zone because it could lead to smaller contact area of the ultrasonic wedge with the weld zone (where the complex acoustic properties are existed). Furthermore, employing the statistical methods (like Taguchi method or ANOVA analysis) to optimize the welding pool and decreasing the HAZ and weld dimensions (similar to works presented by Anawa and Olabi [36-39]) could also be useful to reach more accurate ultrasonic measurements.

\section{Conclusions}

The main goal of this study is evaluating longitudinal residual stress through the thickness of aluminum plates with $8 \mathrm{~mm}$ thickness. Finite element welding simulation along with the $L_{C R}$ ultrasonic method is employed to reach this goal. According to the achieved results, it can be concluded that:

The peak of longitudinal residual stress is (1 occurred in the advancing side (AS) of FSW. The position of peak could be determined by employing the $L_{C R}$ ultrasonic method. Ultrasonic measurement of residual stresses is (2 validated with finite element simulation of FSW process while an acceptable agreement is observed. The little difference between surface and bulk

residual stresses (less than $20 \mathrm{MPa}$ ) could be measured by using different test frequencies in the ultrasonic measurement. This shows relatively high resolution of ultrasonic method in throughthickness stress measurement of aluminum plates. According to the results of this study, simultaneous using of finite element welding simulation and $L_{C R}$ method (known as $F E L_{C R}$ method) can evaluate through-thickness distribution of longitudinal residual stress in FSW process of aluminum plates. The FELCR method could also be 
considered as a nondestructive method for throughthickness stress measurement of materials.

6.

7. References

Rossini NS, Dassisti M, Benyounis KY, Olabi AG. Methods of measuring residual stresses in components. J Materials \& Design 2012; 35:572-88. Olabi AG, Hashmi MSJ. The effect of post-weld

heat-treatment on mechanical-properties and residual-stresses mapping in welded structural steel. Journal of Materials Processing Technology 1995; 55: 117-22. Olabi AG, Hashmi MSJ. The Microstructure and Mechanical Properties of Low Carbon Steel Welded Components After the Application of PWHTs. Journal of Materials Processing Technology 1996; $56: 88-97$.

Olabi AG, Hashmi MSJ. Stress Relief Procedures [4] for Low Carbon Steel (1020) Welded Components. Journal of Materials Processing Technology 1996; $56: 552-62$. Olabi AG, Hashmi MSJ. Effects of the stress- [5] relief conditions on a martensite stainless-steel welded component. Journal of Materials Processing Technology 1998; 77: 216-25. Olabi AG, Casalino G, Benyounis KY, Rotondo A. [6] Minimisation of the residual stress in the heat affected zone by means of numerical methods. Materials and Design 2007; 28: 2295-302. Thomas WM, Nicholas ED, Needham JC, Murch MG, [7] Templesmith P, Dawes CJ. Friction Stir Welding. International Patent Application No. 
PCT/GB92102203 and Great Britain Patent

Application No. 9125978.8, 1991.

Rhodes CG, Mahoney MW, Bingel WH, Spurling RA,

Bampton CC. Effects of friction stir welding on microstructure of 7075 aluminum. Scripta Materialia 1997; 31: 69-75.

Liu HJ, Fujii H, Maeda M, Nogi K. Tensile properties and fracture locations of frictionstir-welded joints of 2017-T351 aluminium alloy. Journal of Materials Processing Technology 2003;

142: $692-6$.

Guerra M, Schmidt C, McClure JC, Murr LE, Nunes [10] AC. Flow patterns during friction stir welding. Materials Characterization 2002; 49: 95-101. Bussu G, Irving PE. The role of residual stress [11] and heat affected zone properties on fatigue crack propagation in friction stir welded 2024T351 aluminium joints. Int J Fatigue 2003; 25: $77-88$.

Peel M, Steuwer A, Preuss M, Withers PJ. [12] Microstructure, Mechanical properties and residual stresses as a function of welding speed in alluminium AA5083 friction stir welds. Acta Mater 2003; 51: 4791-801.

Staron P, Kocak M, Williams S. Residual stresses in friction stir welded Al sheets. Appl Phys A: Mater Sci Process 2002; 74: 1161-2. Preve'y P, Mahoney M. Improved fatigue perform [14] of friction stir welds with low plasticity burnishing: residual stress design and fatigue perform assessment. Mater Sci Forum 2003; 426: 2933-40.

Crecraft DI. The Measurement of Applied and [15] Residual Stresses in Metals Using Ultrasonic Waves. J Sound Vib 1967. 5: 173-92. 
Egle DM, Bray DE. Measurement of Acoustoelastic [16] and Third-Order Elastic Constants for Rail Steel.

J Acoust Soc Am 1976. 60: 741-4.

Tang W, Bray DE. Stress and Yielding Studies [17] Using Critically Refracted Longitudinal Waves. NDE Engineering Codes and Standards and Material Characterization, Montreal: ASME Pressure Vessels Piping Conference, 1996; 322: 41-8.

Bray DE, Tang W. Subsurface stress evaluation [18] in steel plates and bars using the LCR ultrasonic wave. Nuclear Engineering and Design 2001; 207: $231-40$.

Belahcene F, Lu J. Determination of residual [19] stress using critically refracted longitudinal waves and immersion mode. The Journal of Strain

Analysis for Engineering Design 2002; 37: 13-9. Palanichamy $P$, Vasudevan $M$, Jayakumar $T$. [20] Measurement of residual stresses in austenitic stainless steel weld joints using ultrasonic technique. Science and Technology of Welding and Joining 2009; 14: 166-71.

Javadi $Y$, Akhlaghi M, Najafabadi MA. Using [21] Finite Element and Ultrasonic Method to Evaluate Welding Longitudinal Residual Stress through the Thickness in Austenitic Stainless Steel Plates. J Materials and Design 2013; 45: 628-42. Javadi Y, Najafabadi MA, Akhlaghi M. Residual [22] Stress Evaluation in Dissimilar Welded Joints Using Finite Element Simulation and the LCR Ultrasonic Wave. Russian Journal of Nondestructive Testing 2012; 48: 541-52. Javadi Y, Ahmadi Najafabadi M. Comparison [23] between contact and immersion ultrasonic method to evaluate welding residual stresses of 
dissimilar joints. Materials and Design 2013; 47:

$473-82$.

Javadi Y, Afzali O, Raeisi MH, Najafabadi MA. [24]

Nondestructive Evaluation of Welding Residual Stresses in Dissimilar Welded Pipes. Journal of Nondestructive Evaluation 2013; 32: 177-87. Javadi Y, Pirzaman HS, Raeisi MH, Najafabadi [25] MA. Ultrasonic Evaluation of Welding Residual Stresses in Stainless Steel Pressure Vessel. Journal of Pressure Vessel Technology; DOI: $10.1115 / 1.4023432$.

Javadi Y, Pirzaman HS, Raeisi MH, Najafabadi [26] MA. Ultrasonic inspection of a welded stainless steel pipe to evaluate residual stresses through thickness. Materials and Design 2013; 49: 591601.

Javadi Y, Najafabadi MA, Akhlaghi M. Comparison [27] between Contact and Immersion Method in Ultrasonic Stress Measurement of Welded Stainless Steel Plates. Journal of Testing and Evaluation 2013; 41: 1-10.

[28] DEFORM, Deform-3DTM, User manual, 2003.

[29] Buffa G, Hua J, Shivpuri R, Fratini L. A continuum based FEM model for friction stir welding-model development. Mater Sci Eng A 2006; 419: 389-96.

[30] Buffa G, Fratini L, Pasta S, Shivpuri R. On the thermo-mechanical loads and the resultant residual stresses in friction stir process operations. Ann CIRP 2008; 57/1: 287-90.

[31] Buffa G, Ducato A, Fratini L. Numerical procedure for residual stresses prediction in friction stir welding. Finite Elements in Analysis and Design 2011; 47: 470-6.

[32] ABAQUS. Analysis User's Manual 6.11 2011. Vol. I-III. ABAQUS, Inc.

[33] Eltawahni HA, Olabi AG, Benyounis KY. Effect of process parameters and optimization of $\mathrm{CO} 2$ laser cutting of ultra high-performance polyethylene. Materials and Design 2010; 31: 4029-38.

[34] Asadi P, Mahdavinejad RA, Tutunchilar S. Simulation and experimental investigation of FSP of AZ91 magnesium alloy. Materials Science and Engineering A 2011; 528: 6469-77. 
[35] Woo W, Choo H, Brown DW, Feng Z, Liaw PK. Angular distortion and throughthickness residual stress distribution in the friction-stir processed 6061-T6 aluminum alloy. Materials Science and Engineering A 2006; 437: 64-9.

[36] Anawa EM, Olabi AG. Effect of laser welding conditions on toughness of dissimilar welded components. J Appl Mech Mater 2006; 5-6: 375-80.

[37] Anawa EM, Olabi AG. The application of the hole drilling method to define the residual stress of dissimilar laser welded components. J Appl Mech Mater 2007; 7-8: 133-8.

[38] Anawa EM, Olabi AG. Using Taguchi method to optimize welding pool of dissimilar laser-welded components. J Opt Laser Technol 2008; 40: 379-88.

[39] Anawa EM, Olabi AG. Control of welding residual stress for dissimilar laser welded materials. Journal of materials processing technology 2008; 204: 22-33.

8. 
9. List of table captions

Table 1. Acoustoelastic constant $(L)$ measurement 


\section{List of figure captions}

Fig. 1. Basic finite element model (a: Rigid-viscoplastic material model using the DEFORM software; b: Elastoplastic material model using the ABAQUS software)

Fig. 2. Friction stir welding of aluminum plates

Fig. 3. The $L_{C R}$ measurement devices

Fig. 4. Hole-drilling setup on aluminium plates

Fig. 5. Employing the $L_{C R}$ waves and FEM to evaluate through thickness stresses of FSW

Fig. 6. Verification of FE model by employing hole-drilling measurement of longitudinal residual stresses

Fig. 7. Finite element results of longitudinal residual stresses according to the penetration depths of ultrasonic waves

Fig. 8. Finite element and $5 \mathrm{MHz}$ ultrasonic results related to the longitudinal residual stresses

Fig. 9. Finite element and $4 \mathrm{MHz}$ ultrasonic results related to the longitudinal residual stresses

Fig. 10. Finite element and $2 \mathrm{MHz}$ ultrasonic results related to the longitudinal residual stresses

Fig. 11. Finite element and $1 \mathrm{MHz}$ ultrasonic results related to the longitudinal residual stresses

11.

12.

13. 